\title{
Capacity of MIMO Laser Optical (VLC) Wireless Networks
}

\author{
Si Ming Yang ${ }^{1}$, Peng Xue ${ }^{2}$, Bi Cheng $\mathrm{Li}^{1}$, Xian Feng Wang ${ }^{1}$ and Hai Cheng Liu ${ }^{1}$ \\ ${ }^{1}$ Information Science and Technology Institute, Zhengzhou, China \\ ${ }^{2}$ Naval Aeronautical and Astronautical University, Yantai, China \\ "Corresponding Author: Xianfeng Wang (Email:nuaa001@126.com)
}

Keywords: Capacity; MIMO; Laser optical; Wireless network

\begin{abstract}
In wireless communication systems, the laser optics wireless communication systems becomes more and more popular, such as the visual light communication (VLC) system. In this paper, we have analyzed the capacity of MIMO VLC communication system using Shannon Capacity in general, and provide necessary comments on the VLC system. Simulation results has been given to prove the effectiveness and robust of the MIMO VLC system.
\end{abstract}

\section{Introduction}

With rapid development of wireless communication systems, the demand for transmission speed and QoS is more and more severe according to recent researches [1]. Visible light communication (VLC) is a new type of wireless communications using visible light between 400 and $800 \mathrm{THz}(780-375 \mathrm{~nm})$ [2]. The VLC system is suitable to provide high speed transmission with low power consumption and lower interferences.

Researches about VLC recently are very popular. Orthogonal frequency division multiplexing (OFDM) systems used in a VLC will have high peak-to-average power ratios (PAPRs). Since the transmitting LED is limited, peaks of the OFDM symbol can be added to reduce signal reduction. This characteristic also cause in RF communication system [3], although in RF systems it is not to enhance the throughput in charge of RF [4] power consumption effectively. Results on verifying VLC performance are severe [5]. Specifically, VLC is not aware from RF in system constraint, BB signal, and nonlinearity characteristic of the transmitter, it is not obvious how the system is related to communication conversion efficiency in VLC. However VLC suffers from serious drawbacks which decrease the performance. One of the major problems faced by any VLC [6] system is the interference caused by ambient light noise, so in this work, we are trying to evaluate the capacity [7].

The rest of the paper is organized as follows. In part 2, the theoretical analysis is given and in part 3 , the simulation and analysis is given to prove the idea of this work.

\section{Theoretical Analysis}

Assume that in a MIMO system, the sender has $M$ antennas, the receiver has $N$ antennas, the transmitter does not know the channel state information, and the total transmit power $P$.

By the interference of additive white Gaussian noise (AWGN) channel [8], and each receiving antenna noise power is $\sigma^{2}$, so each receiving antenna on the signal-to-noise ratio (SNR) as follows: $\xi=p / \sigma^{2}$.It is assumed that the transmitted signal bandwidth is narrow enough, the channel frequency response can think is flat, with the $M \times N$ complex matrix $H$ to said channel matrix. The first $i j$ elements $h_{i j}$ said first $i$ root launch antenna to the first $j$ root receiving antenna of channel fading coefficient.

Gives the expression of the channel capacity of MIMO systems.

Assumes that each antenna transmit power is $P / M$, and analyze the number of antennas on the channel capacity, and the channel capacity in the situation of a large number of antennas, required to give a theoretical analysis and simulation results. 
Analyze the impact of different power allocation strategies on the channel capacity, required to give a theoretical analysis and simulation results.

\section{Channel capacity derivation}

Set the input signal $x(t)$, the corresponding output signal $y(t)$ to represent. Let $x(t) y(t)$ sample from the signal $x, y$, where $x$ is the $i^{\text {th }}$ element ix express the signals transmitted by the $i^{t^{t h}}$ antenna, the $j^{\text {th }}$ element of $y$, which express the signals received by the $j^{\text {th }}$ antenna, the channel matrix is expressed by the $M \times N$ complex matrix $H$, thus, $y=H x+n, n$ represents the channel additive white Gaussian noise. For simple purposes of the calculation, let SVD decomposition on $H$. Modern signal processing theory shows that: $y=H x+n=U\left(\begin{array}{cc}\operatorname{diag} & 0 \\ 0 & 0\end{array}\right) V^{\mathrm{H}} x+n \cdot U$ and $V$ are non-negative square root of the eigenvalue. MIMO system in the transmitter and the receiver side using multiple antennas and multiple channels, as shown:

Transmission of information flow $S(k)$ the formation of $M$ information sub-streams encoded after the space-time $M$ sub streams sent by the $M$ antennas after spatial channel from $N$ receive antennas to receive multi-antenna receiver is able to take advantage of the advanced encoding processing techniques to separate and decode these data sub-streams, in order to achieve the best treatment. MIMO is using multiple antennas to send and receive at both ends, corresponds to a MIMO sub channel between each transmit and receive antennas, the channel matrix $H$ formed between the transmitting and receiving antennas in time $t$, the channel matrix as:

$$
H(t)=\left[\begin{array}{ll}
h_{1,1}^{t} & h_{2,1}^{t} \ldots . . h_{M, 1}^{t} \\
h_{1,2}^{t} & h_{2,2}^{t} \ldots . . h_{M, 2}^{t} \\
\ldots & \ldots \\
h_{1, N}^{t} & h_{2, N}^{t} \ldots . . h_{M, N}^{t}
\end{array}\right]
$$

Among them, the elements of $\mathrm{H}$ is the gain of any pair of send and receive antenna.

$M$ Sub-streams at the same time sent to the channel, the transmit signals occupy the same band, which did not increase the bandwidth. If the channel response between each transmit antenna is independent, the MIMO system can create multiple parallel spatial channels. Through the parallel channel independent transmission, it will certainly improve the data transfer rate. MIMO channel for the channel matrix parameters, assuming the emission end of the total transmit power $\mathrm{P}$, independent of the number $M$ of the transmitting antenna; for the receiver noise matrix $n$, its elements are independent zero-mean Gaussian complex variable, receiving antenna noise power are $\sigma^{2}, \rho$ is the average signal to noise ratio of the ground. At this point, the transmitted signal is independent M-dimensional statistics, the same energy, the Gaussian distribution of complex vector. The average transmit power allocated to each antenna, the capacity formula is:

$$
C=\log _{2}\left[\operatorname{det}\left(I_{N}+\frac{\rho}{M} H H^{H}\right)\right]
$$

Fix $M, N$ increases, let $\frac{1}{M} H H^{\mathrm{H}} \rightarrow I_{N}$, then can get to the capacity of the approximate expression: $C=N \log _{2}(1+\rho)$.

In equation (2), det represents the determinant, $I_{N}$ represents $M$ dimensional unit matrix, $H^{H}$ represents the conjugate transpose of $H$. From the type can be seen, the channel capacity with the antenna number increases linearly with increasing. That you can use the MIMO channel greatly increase the capacity of wireless channel, without increasing the bandwidth and antenna transmitting power condition, the spectrum utilization rate can be enhanced greatly, fully shows the great 
superiority of MIMO technology.

When the receiver know the channel state information, while, at the transmitter it is unknown, we can make the following assumptions:

Each path independent of each other, and is independent of synchronization in Rayleigh fading channel.

The signal for narrow-band signals, frequency selective fading. (Otherwise consider coding and equalization algorithm)

The channel is static, i.e. the channel matrix $H$ in a burst frame remains constant, while between frames is random variable.

\section{Simulation and Analysis}

When each antenna transmit power is $P / M$, that when transmitter gives average power allocations, you can prove that the optimal covariance matrix:

$$
R_{x}=\left(\frac{P}{M}\right) I_{M}
$$

And the capacity of channel is:

$$
C=\log \operatorname{det}\left(I_{M}+\frac{S N R}{M} H H^{T}\right)
$$

By the Jensen inequality shows: when $Q=\left\{\begin{array}{c}H^{T} H, N<M \\ H H^{T}, N>M\end{array}\right.$

$$
\sum_{\mathrm{i}=1}^{\mathrm{L}} \log \left(1+\frac{S N R}{M} l_{i}^{2}\right) \leq \operatorname{Llog}\left(1+\frac{S N R}{M}\left(\frac{1}{L} \sum_{\mathrm{i}=1}^{\mathrm{L}} \lambda_{\mathrm{i}}^{2}\right)\right)
$$

In theory, when the sender and receiver are aware of the CSI, the injection power allocation is the best allocation of power, but when the transmitter does not know CSI, the optimal covariance matrix is the unit matrix, leading to a different characteristic pattern of distribution of power.

When the transmitter does not know channel but the channel is fully random, optimal covariance matrix is unit matrix, leading to a different characteristic pattern of distribution of the same power then the channel capacity is: $C=\sum_{\mathrm{i}=1}^{\mathrm{L}} \log \left(1+\frac{S N R}{M} \lambda_{\mathrm{i}}^{2}\right)$, That is, when the channel matrix is random matrix, the channel capacity can reach a maximum, random encoding can.

When the transmitter is known to parameters of the channel or channel estimation, the water-filling algorithm can be used to improve performance, to enhance the capacity of the channel. $C=\sum_{\mathrm{i}=1}^{\mathrm{n}} \log \left(1+\frac{P i}{\delta^{2}} \lambda_{\mathrm{i}}^{2}\right)$, every sub channel power sum is equal to the signal power of sending.

The following assumes that the receiver is known to channel $\mathrm{h}$ (receiver channel information can be got through the detection and tracking). $\mathrm{H}$ is random, but you can first consider the simplest case, $\mathrm{h}$ is determined, which is a sample of the channel capacity. To achieve capacity, $\mathrm{s}$ is the complex Gaussian distribution [42]. The corresponding mutual information is:

$$
I=\log _{2} \operatorname{det}\left(\boldsymbol{I}_{M_{R}}+\frac{\boldsymbol{h} \boldsymbol{R}_{s s} \boldsymbol{h}^{H}}{\sigma^{2}}\right)
$$

MIMO channel capacity is:

$$
C=\max _{\boldsymbol{R}_{s s}} \log _{2} \operatorname{det}\left(\boldsymbol{I}_{M_{R}}+\frac{\boldsymbol{h} \boldsymbol{R}_{s s} \boldsymbol{h}^{H}}{\sigma^{2}}\right)
$$

Transmitter channel state information is known in the real system is difficult to achieve. The transmitter channel state information, can only choose white $\mathrm{s}$ in space, that is, Rss $=(\mathrm{PS} / \mathrm{MT})$. This 
means that the transmission of signals from different antennas is independent of power, which is: $I_{C U}=\log _{2} \operatorname{det}\left(\boldsymbol{I}_{M R}+\frac{P_{\mathrm{S}}}{M_{T} \sigma^{2}} \boldsymbol{h h}^{H}\right)$ It can be decomposed into $I_{C U}=\sum_{i=1}^{r} \log _{2}\left(1+\frac{P_{\mathrm{S}}}{M_{T} \sigma^{2}} \lambda_{i}\right)$

Where $\mathrm{r}$ is the rank of $h, \lambda_{i}$ is a positive eigenvalue of $H H^{H}$. Clearly the $I_{C U} \leq C$. Expressed as the spectral efficiency of MIMO channel is $\mathrm{r}$ channel gain, respectively, is $(i=1,2, \cdots, \mathrm{r})$ transmit power is the PS / MT SISO channel. Multi-level spatial data channels between the transmitter and receiver than SISO systems bring big performance gain. For example, the (higher transmit power) transmit power for each additional 3dB, the ICU to increase the $r$ bit / s / Hz; SISO channel capacity increase $1 \mathrm{bit} / \mathrm{s} / \mathrm{Hz}$. If the transmitter is known to channel information, independent of the spatial channel model can send and receive-side linear processing access, transmit power through optimal allocation of water injection model, so that we can achieve the maximum mutual information and capacity C [9] [10].

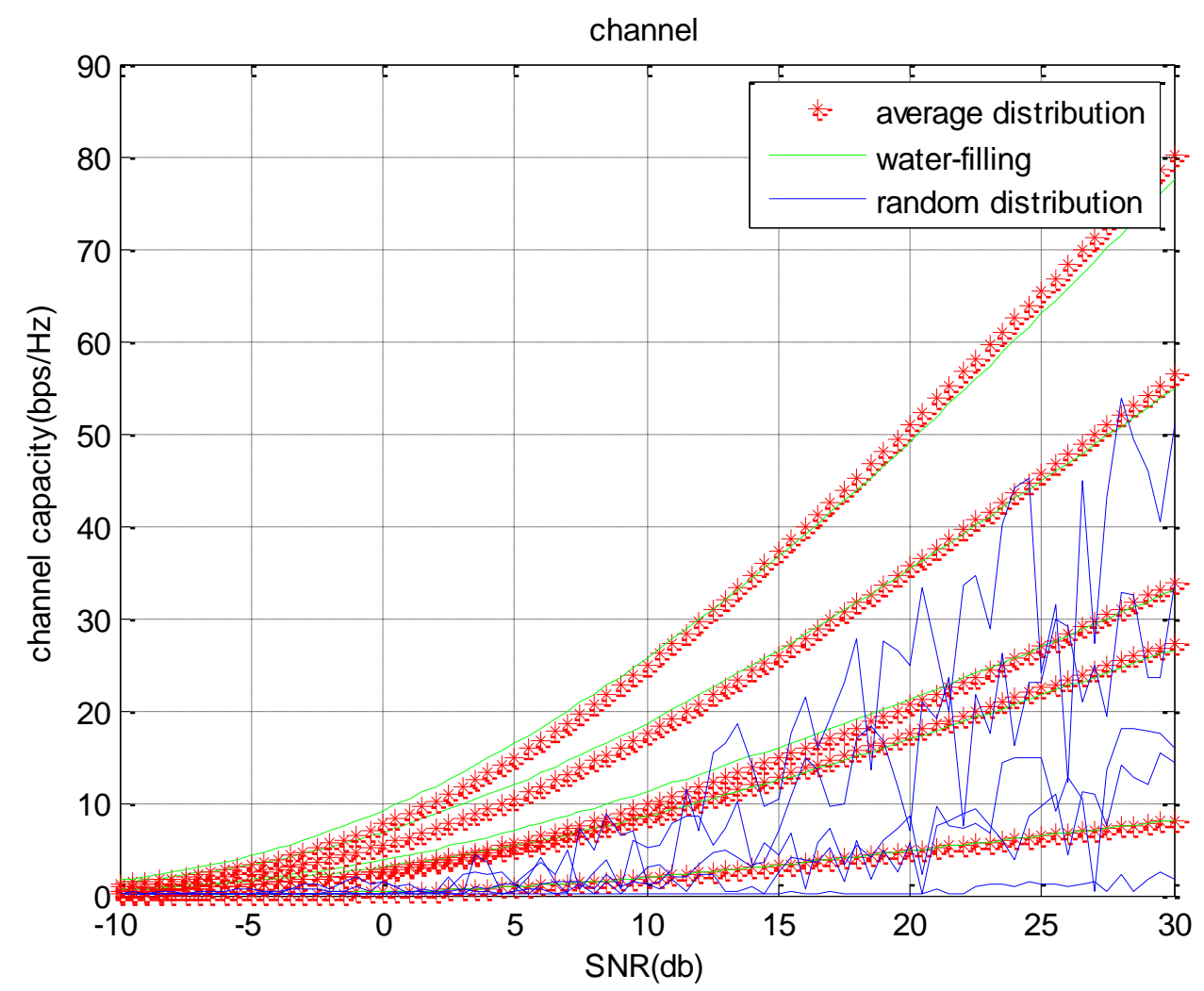

From top to bottom, respectively, $10 \times 10,8 \times 8,6 \times 6,4 \times 4,2 \times 2,1 \times 1$ antenna, can clearly be seen from the figure, the number of antennas increase in channel capacity is obvious; Second, the use of water injection algorithm, the equivalent of full use of channel information, and therefore more able to close the channel capacity; again, in the random coding mode, equivalent to channel a priori knowledge, so the channel capacity is relatively poor.

\section{Summary}

In this paper, we have proposed the capacity of visual light communication system with multiple antennas using Shannon Capacity, the result implies the fact that the system is suitable to provide higher transmission speed with lower cost in fact. 


\section{References}

[1] Sengunthar, A.; Patil, R.; Bhosale, S.; Khodaskar, R.; Talbar, S.N., "Visual Light Communication Using LED," India Educators' Conference (TIIEC), 2013 Texas Instruments , vol., no., pp.121,124, 4-6 April 2013.

[2] Higgins, M.D.; Green, R.J.; Leeson, M.S., "Optical Wireless for Intravehicle Communications: A Channel Viability Analysis," Vehicular Technology, IEEE Transactions on, vol.61, no.1, pp.123,129, Jan. 2012

[3] Shuze Zhao; Jiale $\mathrm{Xu}$; Trescases, O., "A dimmable LED driver for visible light communication (VLC) based on LLC resonant DC-DC converter operating in burst mode," Applied Power Electronics Conference and Exposition (APEC), 2013 Twenty-Eighth Annual IEEE ,pp.2144,2150, 17-21 March 2013

[4] Bhalerao, Manoj V.; Sonavane, S.S., "Visible light communication: A smart way towards wireless communication," Advances in Computing, Communications and Informatics (ICACCI, 2014 International Conference on , pp.1370,1375, 24-27 Sept. 2014

[5] Verma, S.; Shandilya, A.; Singh, A., "A model for reducing the effect of ambient light source in VLC system," Advance Computing Conference (IACC), 2014 IEEE International , pp.186,188, 21-22 Feb. 2014

[6] Zhenhua Yu; Baxley, R.J.; Zhou, G.T., "Peak-to-average power ratio and illumination-to-communication efficiency considerations in visible light OFDM systems," Acoustics, Speech and Signal Processing (ICASSP), 2013 IEEE International Conference on , pp.5397,5401, 26-31 May 2013

[7] Butala, P.M.; Elgala, H.; Little, T.D.C., "SVD-VLC: A novel capacity maximizing VLC MIMO system architecture under illumination constraints," Globecom Workshops (GC Wkshps), 2013 IEEE , vol., no., pp.1087,1092, 9-13 Dec. 2013

[8] Xu Bao; Xiaorong Zhu; Tiecheng Song; Yanqiu Ou, "Protocol Design and Capacity Analysis in Hybrid Network of Visible Light Communication and OFDMA Systems," Vehicular Technology, IEEE Transactions on , vol.63, no.4, pp.1770,1778, May 2014

[9] Jun-Bo Wang; Qing-Song Hu; Jiangzhou Wang; Ming Chen; Yu-Hua Huang; Jin-Yuan Wang, "Capacity analysis for dimmable visible light communications," Communications (ICC), 2014 IEEE International Conference on , pp.3331,3335, 10-14 June 2014

[10]Jun-Bo Wang; Qing-Song Hu; Jiangzhou Wang; Ming Chen; Jin-Yuan Wang, "Tight Bounds on Channel Capacity for Dimmable Visible Light Communications," Lightwave Technology, Journal of , vol.31, no.23, pp.3771,3779, Dec.1, 2013 